\title{
"Round-the-Clock" Surgical Access to the Orbit
}

\author{
Alessandro Paluzzi ${ }^{1}$ Paul A. Gardner ${ }^{1}$ Juan C. Fernandez-Miranda ${ }^{1}$ Matthew J. Tormenti ${ }^{1}$ \\ S. Tonya Stefko ${ }^{3}$ Carl H. Snyderman ${ }^{1,2}$ Joseph C. Maroon ${ }^{1}$ \\ ${ }^{1}$ Department of Neurosurgery, University of Pittsburgh Medical \\ Address for correspondence Alessandro Paluzzi, FRCS (SN), \\ Center, Pittsburgh, Pennsylvania, United States \\ Department of Neurosurgery, Queen Elizabeth Hospital Birmingham, \\ 2 Department of Otolaryngology, University of Pittsburgh Medical \\ Edgbaston, Birmingham, B15 2TH, United Kingdom \\ Center, Pittsburgh, Pennsylvania, United States \\ ${ }^{3}$ Department of Ophthalmology, University of Pittsburgh Medical \\ (e-mail: apaluzzi4@gmail.com).
}

J Neurol Surg B 2015;76:12-24.

\begin{abstract}
Keywords

- orbit

- orbital tumors

- endonasal endoscopic approach

Objective To describe an algorithm to guide surgeons in choosing the most appropriate approach to orbital pathology.

Methods A review of 12 selected illustrative cases operated on at the neurosurgical department of University of Pittsburgh Medical Center over 3 years from 2009 to 2011 was performed. Preoperative coronal magnetic resonance imaging and/or computed tomography views were compared using a "clock model" of the orbit with its center at the optic nerve. The rationale for choosing an external, endoscopic, or combined approach is discussed for each case.

Results Using the right orbit for demonstration of the clock model, the medial transconjunctival approach provides access to the anterior orbit from 1 to 6 o'clock; endoscopic endonasal approaches provide access to the mid and posterior orbit and orbital apex from 1 to 7 o'clock. The lateral micro-orbitotomy gives access to the orbit from 8 to 10 o'clock. The frontotemporal craniotomy with orbital osteotomy accesses the orbit from 9 to 1 o'clock; addition of a zygomatic osteotomy to this extends access from 6 to 8 o'clock.

Conclusions Combined, the approaches described provide 360 degrees of access to the entire orbit with the choice of the optimal approach guided primarily by the avoidance of crossing the plane of the optic nerve.
\end{abstract}

\section{Introduction}

The orbit is a cone-shaped cavity with a quadrangular base and an apex formed by the optic canal and the superior orbital fissure. The optic canal contains the optic nerve and ophthalmic artery; the superior orbital fissure is the gateway for the oculomotor nerves, V1, superior ophthalmic vein, and sympathetic fibers from the cavernous sinus.

Pathologies within the orbit can be classified as intraconal or extraconal based on their relationship with the extraocular muscle cone. Intraconal lesions tend to present with early vision loss, impairment of ocular motility, and axial proptosis.

received

March 14, 2013

accepted

October 10, 2013

published online

September 2, 2014

In contrast, extraconal lesions tend to cause proptosis as an early manifestation and vision loss as a late one. Intracanalicular tumors (within the optic canal) are a subgroup of intraconal lesions that cause early vision loss, optic nerve head edema, and appearance of optociliary shunt vessels on the surface of the optic discs, with minimal or no proptosis.

Excision of orbital lesions can often be challenging, requiring the combined expertise of more than one specialist including ophthalmologists, neurosurgeons, and otolaryngologists. The guiding principle for vision preservation when choosing the best approach is to avoid surgical manipulation of the optic or oculomotor nerves. Therefore, orbital

(c) 2015 Georg Thieme Verlag KG Stuttgart · New York
DOI http://dx.doi.org/ 10.1055/s-0033-1360580. ISSN 2193-6331. 
pathologies lateral to the optic nerve should be accessed via lateral orbitotomies and medial pathologies via medial orbitotomies. These medial corridors can be created through external approaches (such as the anterior medial microorbitotomy or transfacial approaches) or via endonasal corridors.

This study presents an algorithm for selecting the most appropriate anatomical corridor to orbital pathologies, selecting from a range of approaches that render the orbit accessible from 360 degrees. ${ }^{27}$

\section{Methods}

The clinical records of 12 selected patients who underwent treatment of orbital pathologies at our institution over a period of 3 years from April 2008 to April 2011 were reviewed. Patients' demographics, symptoms and signs at presentation, and histologic diagnosis were recorded. The location of the lesion was defined as intraconal (within the extraocular muscle cone), extraconal, or intracanalicular (within the optic canal). The patients' preoperative coronal magnetic resonance imaging (MRI) and/or computed tomography scan images were compared using a "clock model" of the right orbit with its center in the optic nerve. For the images to be read in a clockwise direction, the scans of left sided lesions were flipped horizontally so that a mirror image was obtained. The type of approach used was classified as external (frontotemporal craniotomy/orbitotomy with or without zygomatic osteotomy, lateral orbitotomy, anterior medial micro-orbitotomy), endoscopic endonasal, or combined external and endoscopic endonasal. The clinical outcome as well as the radiologic outcome (percentage of lesion removed) was also recorded( - Table $\mathbf{1})$.

\section{Surgical Techniques}

\section{Frontotemporal Craniotomy with Orbitozygomatic Osteotomy}

A curvilinear incision starting just anterior to the tragus up to the midline apex of the anterior hairline (so-called widow's peak) is usually sufficient to access the superolateral orbit (-Fig. 1). However, to extend the approach medially to the superior orbit or inferolaterally, a modified bicoronal incision, from tragus to tragus or to the contralateral superior temporal line, can be used. A differential flap (scalp and temporalis muscle) is developed elevating the fat pad between the superficial and deep layers of the temporalis fascia to protect the frontalis branches of the facial nerve. ${ }^{1}$ Depending on the degree of inferior access required, the zygoma is exposed and the masseter muscle is detached from its inferior border. A subperiosteal dissection should be carried onto the orbit and around its rim, dissecting the periorbita from the inner orbit. The supraorbital neurovascular bundle is either dissected from its notch or freed from its foramen with diagonal osteotomies (inverted V), directed away from the nerve. A frontotemporal craniotomy is performed and the lateral bone of the greater wing of the sphenoid is then removed with rongeurs and drill until it is flush with the orbit. To maximize the amount of orbital roof preservation, it is important to identify, coagulate, and transect the meningo-orbital fold. This provides exposure of the superior orbital fissure (SOF), which is potentially the most posterior limit of the superior orbitotomy.

The frontal dura is then dissected free from the roof of the orbit and the periorbita is dissected from the inner roof. Two malleable retractors are inserted along these planes on either side of the orbital roof while osteotomies are performed with a reciprocating saw. The medial cut is usually at, or just lateral to, the supraorbital notch; the lateral cut is made from the inferior orbital fissure to a level just above (for supralateral orbital osteotomy) or below (for orbitozygomatic osteotomy) the zygomatic prominence. For an orbitozygomatic osteotomy, a posterior cut is also made across the zygomatic arch just anterior to the glenoid fossa. Finally, the osteotomy is completed using a small drill bit from the dural side, protecting the orbit with a malleable retractor, connecting the medial to the lateral osteotomy along the posterior part of the orbital roof. This osteotomy runs from just anterior to the SOF to the inferior orbital fissure (IOF) and aims to preserve most of the orbital roof. ${ }^{2-4}$ Once the orbitozygomatic complex is freed from any remaining soft tissue attachment and removed, access is provided to the entire superolateral orbit to just below the inferior orbital fissure. It is important to complete the bony removal at the posterior portion of the superior orbit because this bone can prevent adequate dural retraction and defeat much of the advantage of the superior orbitotomy.

Intraorbital tumors can be identified with digital palpation, image guidance, or intraoperative ultrasound. Periorbita is opened in an anterior-posterior direction and dissection performed through the periorbital fat. This can be carefully shrunk using bipolar diathermy. Limiting the initial periorbital opening to just over the tumor can limit fat herniation. If there is tumor in the medial or superior intraconal space or affecting the optic nerve, the annulus of Zinn should be opened medial to the levator and superior rectus muscles to prevent injury to the oculomotor nerve. These muscles can be retracted laterally for improved exposure. Caution must be used when retracting these muscles because the innervation to them ramifies and penetrates the muscles on their inner (intraconal) surfaces at approximately the junction of the posterior one third and anterior two thirds. It can be difficult to preserve the trochlear nerve with this approach, but it should be attempted. For these reasons, we limit lateral to medial access to approximately the 1 o'clock position. Standard microsurgical techniques are used for tumor dissection and removal.

\section{Lateral Orbitotomy}

The skin incision used in the lateral orbitotomy approach is a small brow or cantholysis incision (extending from the lateral canthus in a natural skin crease), which is well hidden in patients who wear glasses (-Fig. 2). The anterior margin of the temporalis muscle is dissected from the underlying bone, exposing the lateral wall of the orbit. A subperiosteal dissection of the orbital side of the bone allows retraction of orbital contents including the lacrimal gland. 


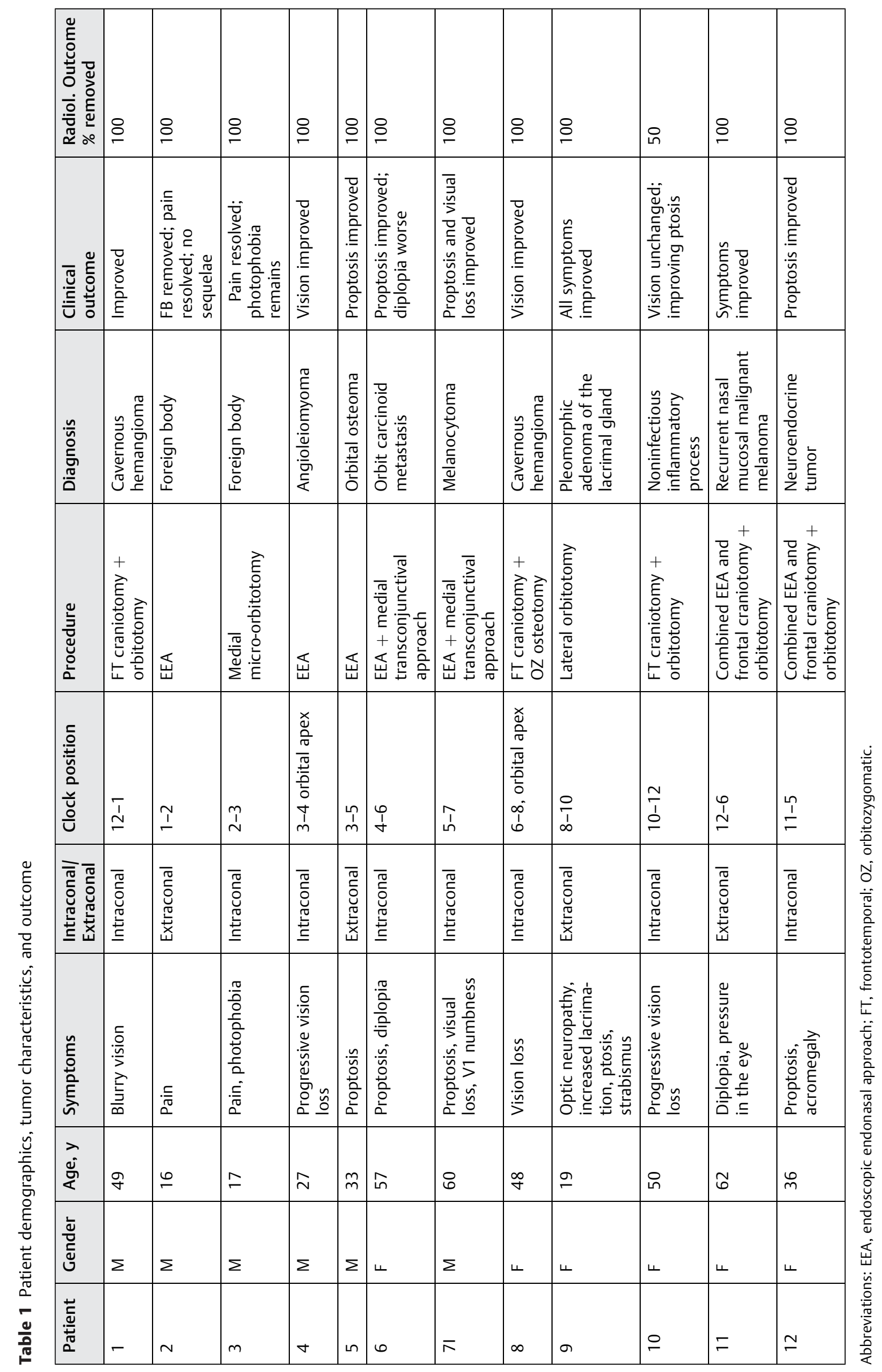



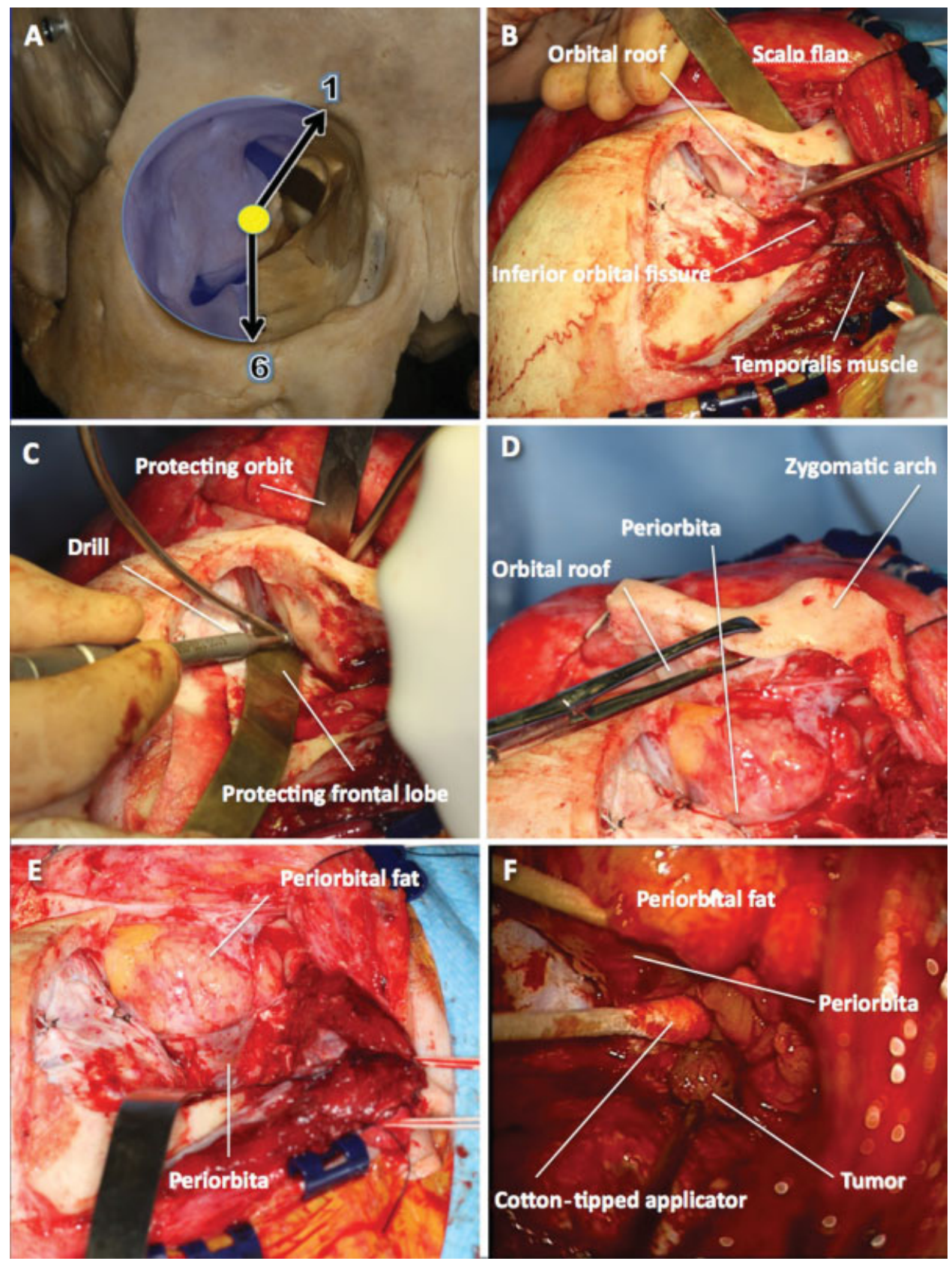

Fig. 1 Frontotemporal craniotomy with orbitozygomatic osteotomy. (A) Clock model showing the extent of the orbit that can be exposed through this approach. (B) The frontotemporal craniotomy (first piece) has been cut, the temporalis muscle dissected off its anterior attachment and retracted posteriorly, and a malleable retractor inserted between the orbital roof and the periorbita. (C) While protecting the orbit content on one side of the orbital roof and the frontal lobe on the other side with malleable retractors, the final cut over the orbital roof is made with a highspeed drill going laterally toward the inferior orbital fissure. (D) The "second piece" of the orbitozygomatic craniotomy is removed exposing (E) periorbita and periorbital fat. (F) After retraction of muscles and periorbital fat with cotton-tipped applicators, the tumor comes into view (photo taken with operative microscope).

Two transverse osteotomies are made in the lateral orbital bone using a reciprocating saw, the first superior to the zygomaticofrontal suture and the second just above the origin of the zygomatic arch. This lateral orbital bone can be drilled, cracked with an osteotome, or fractured posteriorly by grasping the rim with a rongeur. The exposed sphenoid wing is removed with a high-speed drill and rongeurs as needed to reach the level of the orbital apex. The periorbita is opened parallel to the lateral rectus muscle depending on tumor location within the muscle cone. Identification of the lateral rectus muscle in the posterior orbit can prove difficult, so this muscle can be identified at its insertion in the globe and retracted with either a vessel loop or traction suture, helping to improve the lateral access into the orbit at the same time.

If there is optic nerve involvement by the tumor, the optic nerve should be identified proximal and distal to the tumor. As with all approaches, standard microsurgical techniques are used for tumor debulking and resection. 

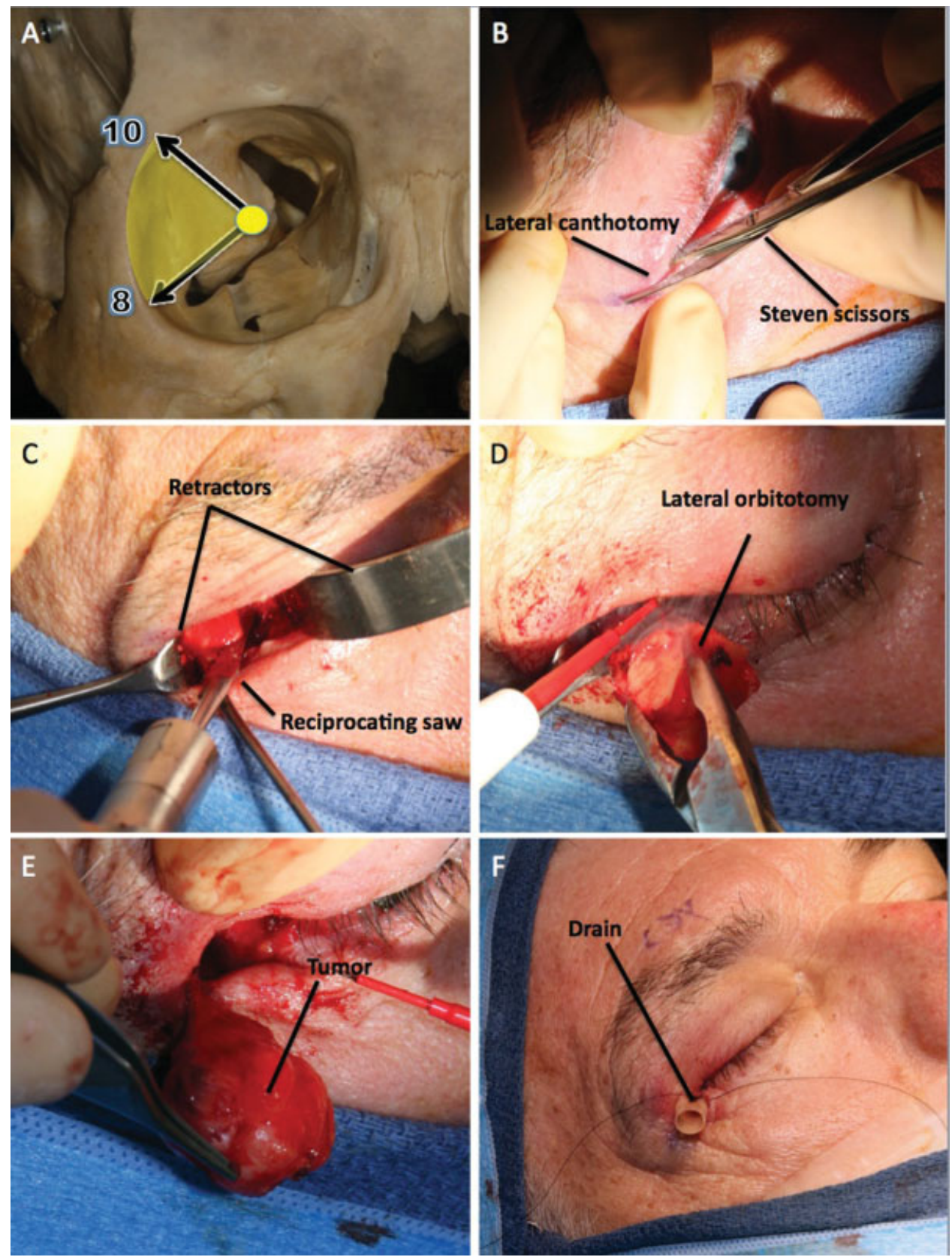

Fig. 2 Lateral orbitotomy. (A) This approach is ideally suited for a lesion lateral to the optic nerve at the 8-10 o'clock position. (B) A small cantholysis incision is made with Steven scissors along a skin crease. (C) The temporalis muscle has been detached and retracted laterally; with adequate retraction it possible to expose the whole lateral wall of the orbit even with a relatively small incision. The osteotomy is performed with a reciprocating saw. (D) The lateral orbitotomy has been completed and the last fibers of the temporalis muscle cut with a monopolar knife. (E) The periorbita has been opened and the tumor completely removed. (F) A soft drain has been secured in place.

\section{Anterior Medial Micro-Orbitotomy}

A medial micro-orbitotomy gives access to tumors located anterior and medial in the orbit (-Fig. 3). Ophthalmic preparation of the orbit and surrounding area is performed, and a small eyelid retractor is placed. A conjunctival peritomy is performed 90 degrees around the cornea. The medial rectus muscle is isolated and controlled with a double-armed suture at its insertion site into the globe after relaxing conjunctival incisions are made superior and inferior to the muscle. The muscle is then freed from its intermuscular septa and distal ligaments are cut from the insertion site. For more inferiorly located lesions, the inferior rectus muscle might need to be retracted or even detached. Handheld retractors can then be used to retract the globe laterally and the medial rectus muscle medially. The orbital fat is dissected under the operating microscope using cottonoids or cotton-tip applicators for orbital fat retraction.

After tumor resection, the medial rectus muscle is reattached at its insertion site on the globe with a $6-0$ absorbable suture, and the conjunctiva is closed with interrupted sutures through the conjunctiva and limbus at the superior and inferior relaxing incisions. 

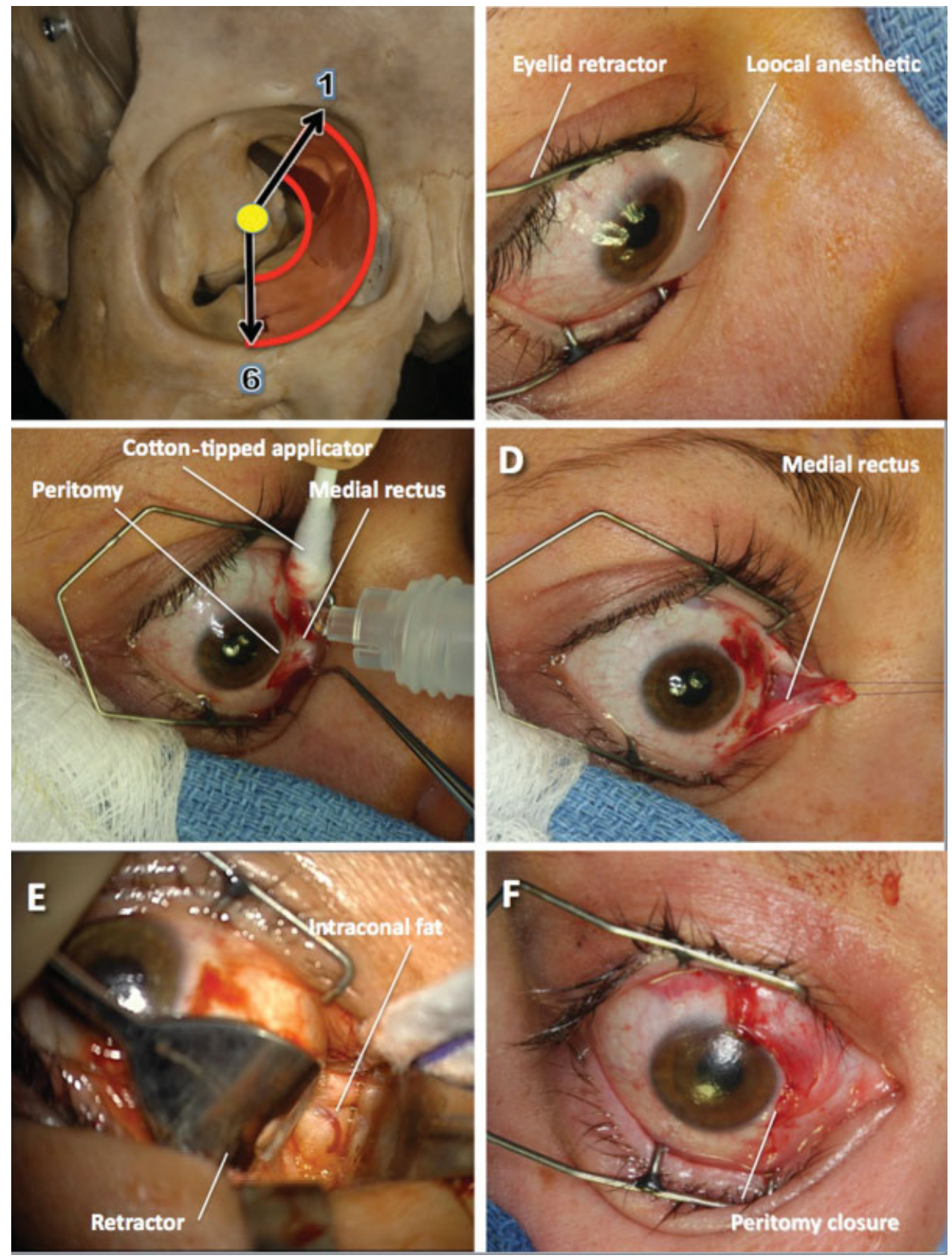

Fig. 3 Medial micro-orbitotomy. (A) This approach gives access to lesions located anterior and medial in the orbit. (B) An eyelid retractor is placed and local anesthetic injected where the peritomy will be performed. (C) After the conjunctiva is incised around the cornea and relaxing conjunctival incisions are made, the medial rectus muscle is detached and (D) retracted medially with a suture. (E) The eye globe is retracted laterally and the intraconal fat exposed. (F) After the lesion has been excised, the medial rectus muscle is reattached at its insertion site on the globe with a 6-0 absorbable suture, and the conjunctiva is closed with interrupted sutures.

\section{Endonasal Approach}

A 0-degree endoscope is used for most of the case, however, a 45-degree endoscope often becomes necessary for working near the orbital apex, particularly inside the muscle conus, or anteriorly in the orbit. The initial steps to expose the entire medial and inferior orbital walls include a complete uncinectomy with wide maxillary antrostomy, anterior and posterior ethmoidectomies, and sphenoidotomy (-Fig. 4). Removal of the orbital floor is limited by the course of the infraorbital nerve. A uninarial approach with preservation of the middle turbinate can often be used, particularly in the case of small extraconal tumors in the medial orbit. A binarial approach provides more room for manipulating the instruments when dissection of the orbital apex or intraconal work is required. In this case the middle turbinate ipsilateral to the pathology is removed together with the posterior nasal septum, and the sphenoid sinus rostrum is opened widely. The septum is limiting when using the contralateral nostril for introduction of instruments except when working at the orbital apex. To limit septum resection, anterior pathologies are best accessed entirely through the ipsilateral nostril. The lamina papyracea is removed to provide access to the medial orbit, and the optic 

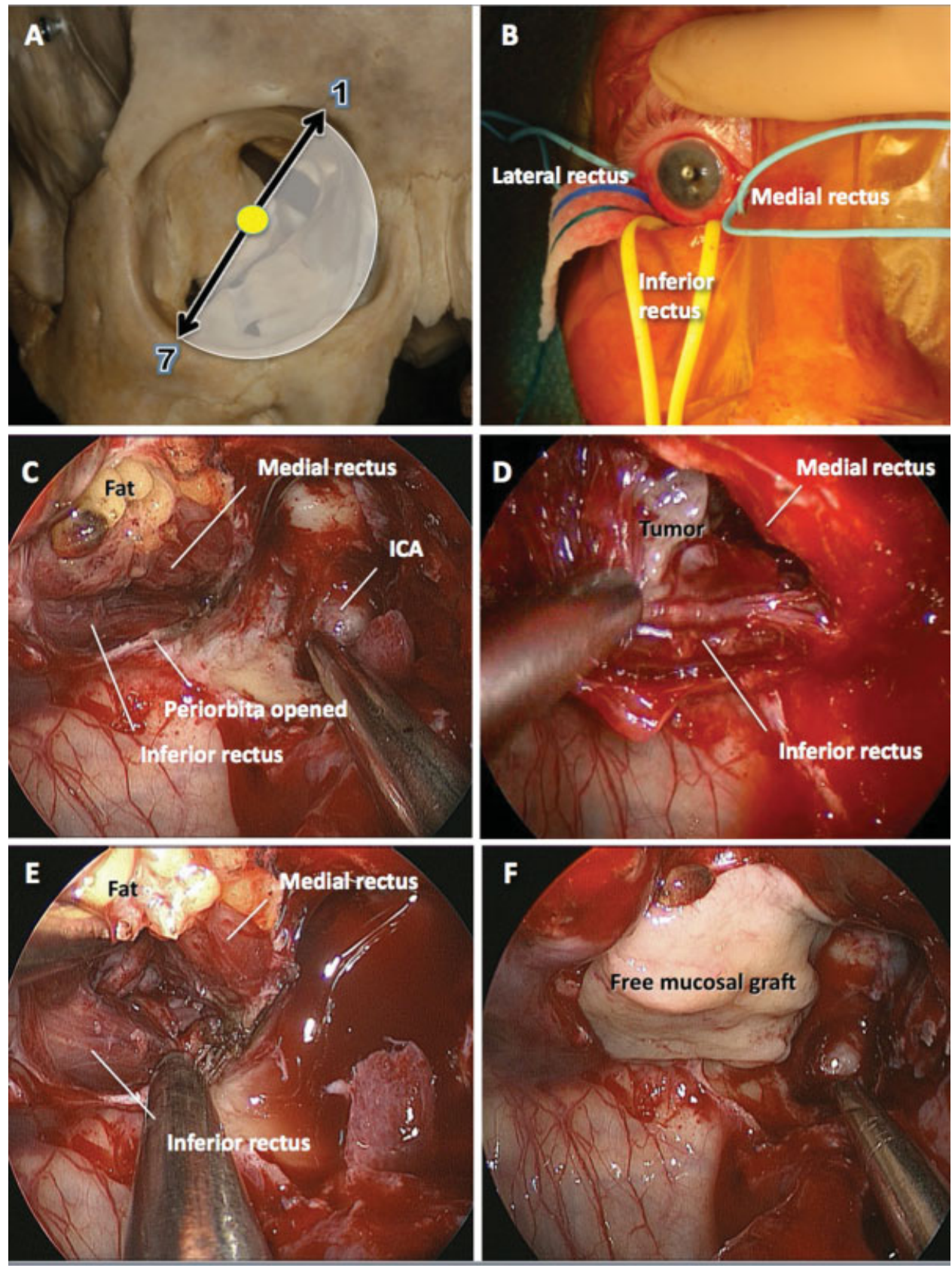

Fig. 4 Endoscopic endonasal approach. (A) Clock model showing the extent of the orbit that can be exposed through this approach. (B) The insertions of the rectus muscles to the globe are identified and controlled with vessel loops. (C) Endoscopic view of the medial aspect of the orbital apex after a portion of the periorbita has been excised. The internal carotid artery (ICA) is visible medially. The window between medial and inferior rectus muscles is "closed." (D) After external retraction on the medial and inferior rectus muscles by pulling the respective vessel loops, the surgical window in now "open" and the tumor is identified and (E) excised. (F) The periorbital defect is covered with a free mucosal graft harvested from the removed ipsilateral middle turbinate.

nerve and carotid artery are identified posteriorly. It is critical to identify the medial and lateral opticocarotid recess which represent the intersection of the optic nerve and internal carotid artery at the opticocarotid cistern and optic strut, respectively.

For inferiorly located (4-7 o'clock) extraconal tumors, a medial maxillectomy is extended anteriorly to give access to the floor of the orbit, inferior rectus muscle, and the orbital contents above. The pterygopalatine fossa is dissected to identify the maxillary branch (2nd division) of the trigeminal nerve to avoid damaging it. If a tumor extends to Meckel cave, it is particularly important to avoid damaging the ophthalmic branch during this dissection because the vidian nerve might have already been injured during the dissection of the pterygopalatine fossa. The loss of corneal sensation together with decreased lacrimation is likely to lead to significant corneal morbidity. In any case, vidian nerve preservation is always attempted. ${ }^{5}$ 
For posterior medial/inferior intraconal lesions, the endonasal dissection corridor is between the medial and inferior rectus muscles. The periorbita is opened parallel to the medial rectus muscle. The extraconal fat can be cauterized with bipolar diathermy if it herniates into the field and the rectus muscles are identified. A cotton-tipped applicator can serve as an excellent retractor of orbital fat endonasally as well. The biggest challenge when working in the intraconal space is keeping the dissection corridor open because the orbital contents are all freely mobile and compressible. This problem can be overcome by working in conjunction with an oculoplastic surgeon who can access and retract the rectus muscles through an external peritomy. The insertions of the rectus muscles to the globe are identified and controlled with vessel loops. This serves two purposes: to aid in the endoscopic identification of the muscles and to provide some anterior muscle retraction and open a window between them toward the orbital apex. In addition, a small ribbon retractor can be introduced to help retract orbital fat. Standard microsurgical techniques are used, bearing in mind that the optic nerve should be lateral and superior to the lesion and the ophthalmic and central retinal arteries course medial to the optic nerve at the orbital apex.

Another way of controlling the rectus muscles includes detaching the medial rectus muscle from the globe, securing it with a silk suture and passing the suture from within the orbit into the nasal cavity. This opens the medial orbit like a book with the medial rectus pedicled posteriorly on the annulus of Zinn. After tumor resection, the rectus muscle is replaced and sutured back onto the globe without loss of function.

We often reconstruct the medial orbital wall using a pedicled nasoseptal mucoperichondrial flap ${ }^{6}$ to prevent excessive scarring around the rectus muscles resulting in restriction of movement and diplopia. Generally, we do not use nasal packing to avoid exerting too much pressure on the globe or optic nerve.

\section{Results}

The results are shown in - Figs. 5 and $\mathbf{6}$.

\section{Patient 1: Intraconal Cavernous Hemangioma, 12-1 O'Clock: Frontotemporal Craniotomy and Orbitotomy} This 48-year-old man developed progressive visual disturbance from a cavernous hemangioma situated between the superior rectus and superior oblique muscles. Its location superior to the optic nerve and almost touching the orbital roof made an orbitofrontal craniotomy the most favorable approach. The lesion was completely removed and the vision in the affected eye improved.

\section{Patient 2: Extraconal Foreign Body, 1-2 0'Clock: Endonasal Endoscopic Approach}

Patient 2 was a 15-year-old boy who fell on a wooden stick that penetrated the anterior cranial fossa passing through his orbit, the lamina papyracea, and the cribriform plate. The patient did not display any neuro-ophthalmologic deficits preoperatively. It was felt that the safest way to "pull" this foreign body out was after visualizing its entire course through an endonasal endoscopic approach (EEA). The distal part of the stick was freed by removing some of the cribriform plate and the lamina papyracea that was entrapping the distal tip. Once loose, it was pulled externally through the orbital entry point. The endoscopic approach also allowed repair of the skull base durotomy and cerebrospinal fluid leak. The patient had intact orbital function postoperatively.

\section{Patient 3: Orbital Foreign Body, 2-3 O’Clock: Medial Micro-Orbitotomy}

This 17-year-old young man was accidentally shot in the orbit by a BB gun. He presented with pain at the extremes of gaze and photophobia but normal visual acuity and visual fields. Because of the relatively anterior location of the foreign body in relation to the orbital apex, it was approached and removed with a medial micro-orbitotomy. His pain improved postoperatively, although at follow-up he still complained of residual photophobia.

\section{Patient 4: Orbital Apex Angioleiomyoma, 3-4 0'Clock: Endonasal Endoscopic Approach}

Patient 4 was a 26-year-old man who presented with progressive visual loss due to compression of the optic nerve by a lesion located on the medial side of the orbital apex. This lesion, due to its proximity to the orbital apex (posterior location), was approached and completely removed through the endoscopic endonasal route. The patient's vision improved postoperatively.

\section{Patient 5: Orbital Osteoma, 3-5 0’Clock: Endonasal Endoscopic Approach}

This 25-year-old man developed progressive proptosis due to a medial orbital osteoma. The EEA was judged to be the most direct and safest route to this lesion. The osteoma was completely excised, leading to complete disappearance of the proptosis without complications.

\section{Patient 6: Intraconal Metastasis, 4-6 0’Clock: Endonasal Endoscopic Approach and Medial Transconjunctival Approach}

Patient 6 was a 57-year-old woman who presented with proptosis, an isolated right inferior rectus deficit, and diplopia. The symptoms failed to respond to steroids and radiotherapy. The lesion was located within the muscle cone in the posterior part of the orbit and appeared to be invading the inferior rectus muscle. The approach began with a medial peritomy from 3 to 7 o'clock through which the inferior rectus was released and the medial rectus retracted with a soft vessel loop. This allowed the complete removal of the lesion under endoscopic visualization through a window inferior to the medial rectus muscle. The proptosis improved after the operation, although the inferior rectus deficit and the diplopia persisted. Histologic examination confirmed this tumor to be a metastatic lesion from an undiagnosed bowel carcinoid that was subsequently treated with surgery and chemotherapy. Patient remained tumor free at 2-year follow-up. 

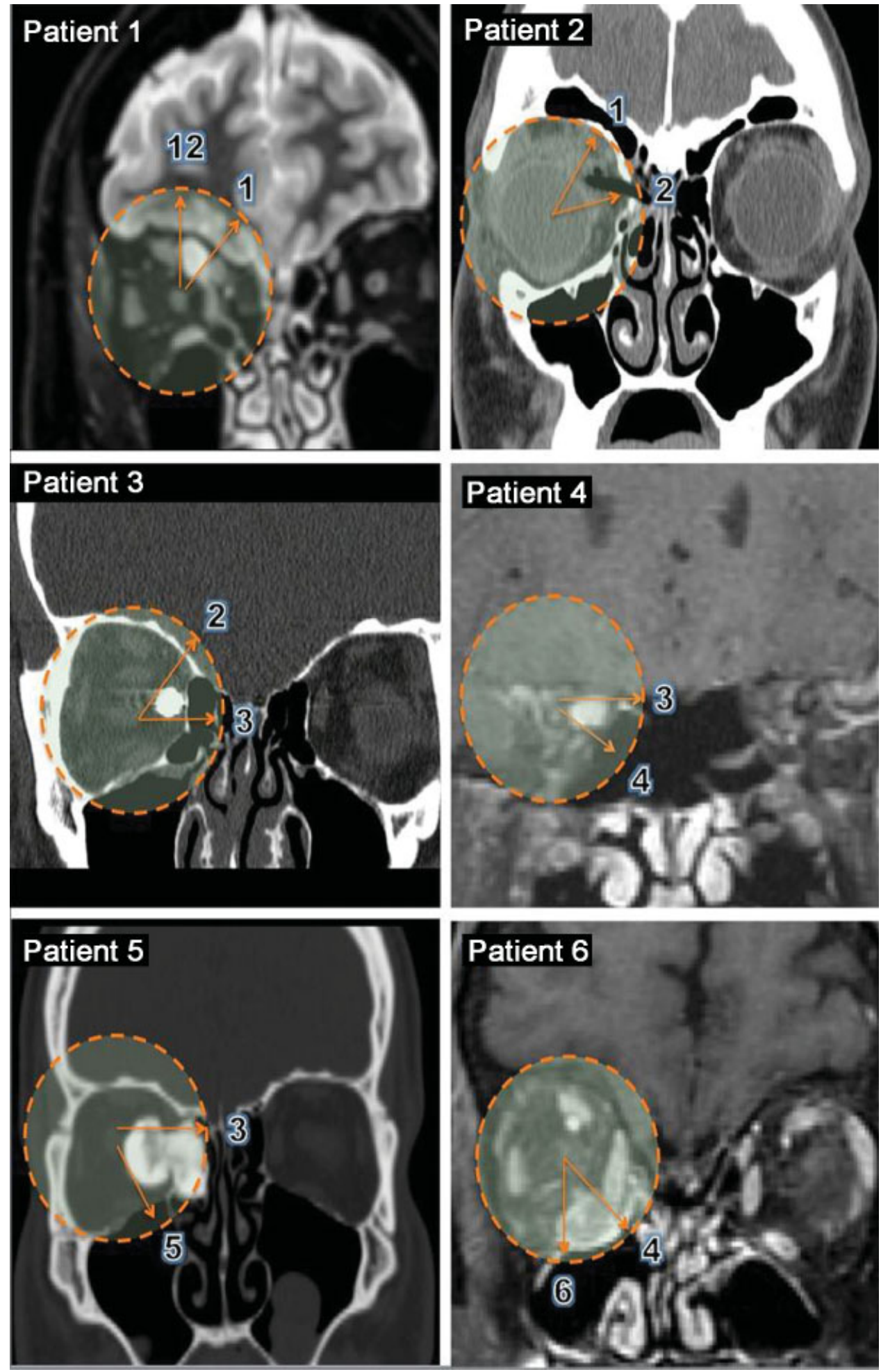

Fig. 5 Coronal preoperative magnetic resonance imaging and computed tomography scans of patients 1 to 6 : 1 , cavernous hemangioma; 2 , foreign body; 3 , foreign body; 4, angioleiomyoma; 5, orbital osteoma; 6 , carcinoid metastasis.

Patient 7: Intraconal Melanocytoma. 5-7 0’Clock: Endonasal Endoscopic Approach and Medial Transconjunctival Approach

This 60-year-old man displayed moderate proptosis, progressive visual deterioration, and numbness over the area of distribution of V1. The radiologic appearance was sug- gestive of a malignant process with lymphoma a strong possibility, and the lesion seemed to involve or abut the inferior rectus muscle. For this reason plus patient comorbidities and tumor location, we decided to approach it endoscopically. Intraoperative frozen section examination showed a spindle cell tumor. We decided to excise the 

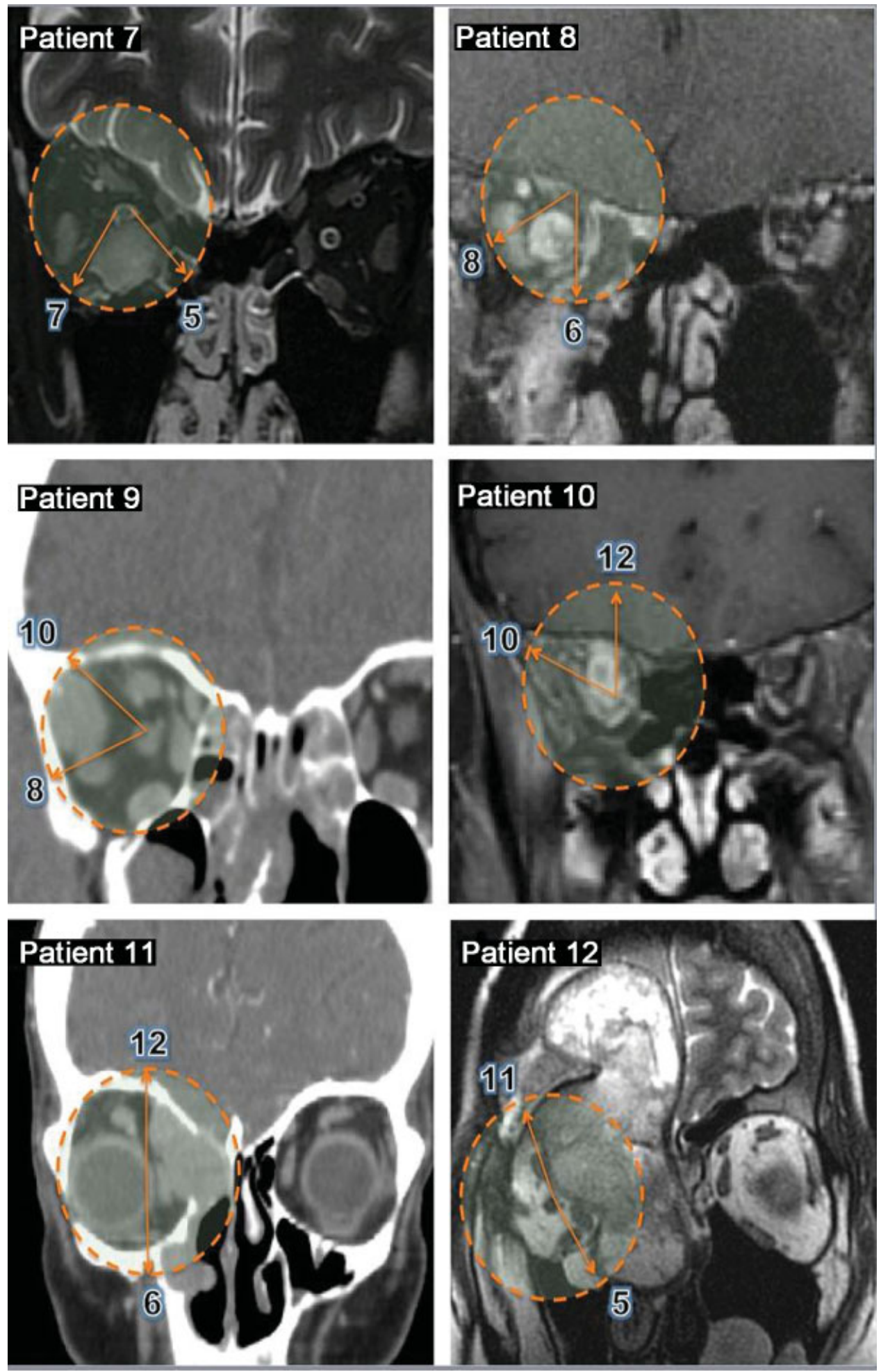

Fig. 6 Coronal preoperative magnetic resonance imaging and computed tomography scans of patients 7 to $12: 7$, melanocytoma; 8, cavernous angioma; 9 , pleomorphic adenoma of the lacrimal gland; 10 , Noninfectious inflammatory process; 11 , recurrent nasal mucosal malignant melanoma; 12, neuroendocrine tumor.

lesion endoscopically with the aid of a medial transconjunctival approach to aid in muscle and fat retraction. The lesion was macroscopically removed, and the patient's vision and proptosis improved postoperatively; the numbness remained. Subsequent histologic examination confirmed the tumor to be a melanocytoma.
Patient 8: Intraconal Cavernous Angioma, 6-8 0'Clock: Frontotemporal Craniotomy and Orbitozygomatic Osteotomy

Patient 8 was a 64-year-old woman complaining of rapidly progressive unilateral vision loss on a background history of blindness in the contralateral eye due to a congenital cataract. 
MRI scan of the orbit showed a cavernous angioma located lateral and inferior to the optic nerve and the ophthalmic artery at the orbital apex. To provide immediate decompression of the optic nerve and minimize the visual risks associated with tumor manipulation, an endoscopic medial orbital apex bony decompression was performed as a first stage. The patient showed complete improvement of her visual field defect, but upon tapering down dexamethasone treatment, the visual defect resumed. A decision was made to remove the lesion via an orbitozygomatic craniotomy. The exposure extended from the IOF to the SOF. The safest window to approach the angioma, to avoid manipulation and potential damage to ophthalmic vessels and oculomotor nerves, was judged to be between the lateral rectus and inferior rectus muscles. The periorbita was opened just superior to the inferior rectus muscle. The short ciliary nerves were attached to the cavernoma and had to be dissected leading to a postoperative transient mydriasis. The cavernoma was completely removed, and the patient regained normal visual function.

\section{Patient 9: Extraconal Pleomorphic Adenoma of the Lacrimal Gland, 8-10 0'Clock: Lateral Orbitotomy}

This 68-year-old man was initially treated by our team for a growth hormone $(\mathrm{GH})$-secreting pituitary adenoma, removed via an endoscopic endonasal approach. He was then noted to have a unilateral mild optic neuropathy with decreased light saturation, increased lacrimation, mild ptosis, and strabismus. An MRI scan of orbit showed a well-circumscribed tumor in the lateral aspect of the orbit, superficial to the window between the superior and lateral rectus muscles, with some degree of calcification suggestive of a benign lesion. Its lateral and superficial location within the orbit made it the perfect case for a lateral orbitotomy. The lesion was completely excised, followed by improvement of all symptoms.

\section{Patient 10: Noninfectious Inflammatory Process, 10-12 O'Clock: Frontotemporal Craniotomy and Orbitotomy}

Patient 10 was a 49 -year-old woman presenting with progressive visual loss and ptosis due to an intraconal mass. Because the lesion was located superior and lateral to the optic nerve near the orbital apex, a craniotomy plus supralateral orbitotomy was selected to take advantage of the window between the lateral and superior rectus muscles. Intraoperative biopsy ruled out a neoplastic lesion; therefore, only partial debulking of the lesion was performed. Postoperatively her vision failed to recover, although the ptosis improved.

Patient 11: Extraconal Recurrent Sinonasal Melanoma. 12-6 O'Clock: Combined Endonasal Endoscopic Approach and Frontal Craniotomy Plus Orbitotomy

A 61-year-old woman presented with diplopia and pressure behind the eye from a recurrent sinonasal melanoma, previously treated endoscopically. Because of the extensive disease and the need for negative dural margins, it was decided to combine the EEA with a frontal craniotomy and orbitotomy.
The entire tumor was removed, and both symptoms improved postoperatively.

Patient 12: Invasive GH-Secreting Pituitary Adenoma, 11-5 0'Clock: Combined Endonasal Endoscopic Approach and Frontal Craniotomy Plus Orbitotomy

This 35-year-old woman previously underwent an endonasal transsphenoidal resection of an invasive GH-secreting adenoma followed by a frontal craniotomy. Within a year she developed progressive proptosis due to a large recurrent tumor extending from the sinonasal cavity to the orbit and anterior cranial fossa. Due to the wide extension of the tumor and to avoid excessive orbital retraction, a combination of frontal craniotomy with orbitotomy and endoscopic endonasal approach was used. The tumor was completely removed and the proptosis improved. $\mathrm{GH}$ and insulinlike growth factor-1 remain within normal limits.

\section{Discussion}

The location of the pathology within the orbit, relative to the optic nerve, should dictate the choice of approach. This is the key guiding principle for orbital approaches. When addressing lesions located superior and lateral to the optic nerve and orbit, traditional neurosurgical approaches like a frontotemporal craniotomy with or without orbitozygomatic osteotomy provide excellent exposure. A lesser variant of this approach is the lateral micro-orbitotomy (as previously described) that is reserved for lesions lateral to the optic nerve and apex. When it comes to pathologies situated very anterior in the orbit and medially, ophthalmologists are more familiar with the anterior medial "orbitotomy," which uses a transconjunctival approach that does not require an osteotomy. ${ }^{7-10}$ This approach, however, is limited to lesions located anterior to the posterior plane of the globe. ${ }^{11}$ When approaching lesions located in the proximity of the orbital apex, the exposure is often significantly limited by the intraorbital soft tissues. A helpful maneuver in these difficult cases involves detaching the medial rectus muscle and mobilizing the cone via a lateral orbitotomy. In spite of this, the surgical field often ends up being a deep cone-shaped area with suboptimal visibility in the depth at the tumor.

Endoscopic assistance through standard external approaches was used to improve visualization as early as the 1980s. Thyroid eye disease and traumatic optic neuropathy not responding to steroids have been successfully treated with endoscopic endonasal orbital and optic nerve decompressions. ${ }^{12-17}$ Reports of orbital tumors that have been biopsied, resected, or decompressed through an EEA have increased in the last few years. ${ }^{18-21}$ The EEA is now a widely accepted approach for the resection of skull base tumors including anterior, middle, and posterior fossae intradural tumors. ${ }^{22-26}$ Recognized advantages are the increased illumination and magnification, the improved cosmesis resulting from the absence of external scars, and shorter hospitalization. Much more importantly, though, the EEA provides unparalleled views of the medial orbital apex and excellent access to intra- and extraconal orbital tumors that are medial 


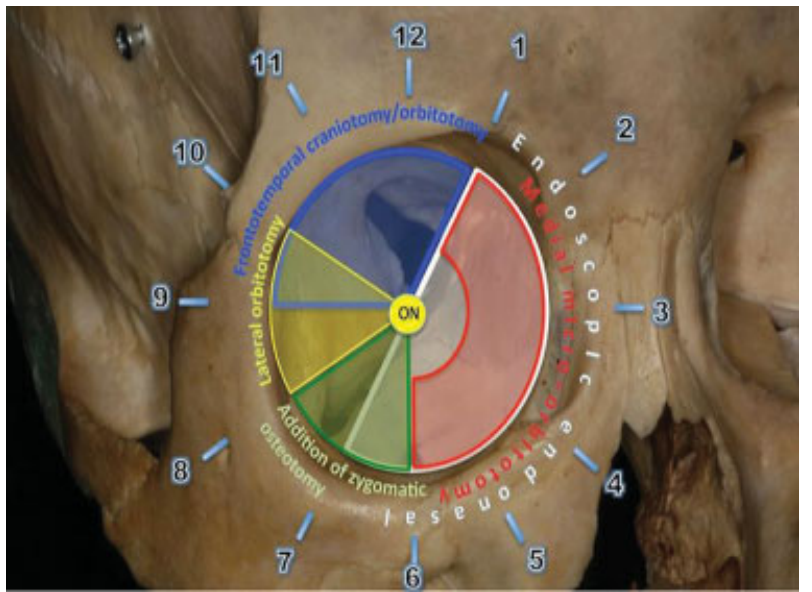

Fig. 7 Clock model of the orbit summarizing how the different approaches fit together and overlap. ON, optic nerve.

and inferior to the optic nerve. It is also particularly useful in the case of medial orbital tumors with medial intracranial extension or for tumors that extend inferior to the optic nerve toward or beyond the orbital floor. For isolated lateral orbital tumors, however, a lateral orbitotomy via a curved hairline incision or a small brow or cantholysis incision is still a far better approach with less morbidity.

Disadvantages of the EEA include nasal morbidity and the need for two experienced endoscopic surgeons familiar with the anatomy of the orbit. In some cases, three surgeons might be required, particularly when dealing with intraconal tumors, typically a neurosurgeon, otolaryngologist, and ophthalmologist. This approach depends on specialized endoscopic instrumentation and angled dissectors. A limitation of the EEA for orbital pathologies is also the absence of effective and nontraumatic endonasal muscle retractors.

Based on our institution's experience with both external and endonasal to orbital pathologies, we designed a simple algorithm that should guide the selection of the most appropriate approach. ${ }^{24}$ As explained earlier, the orbit (right side) is compared with a clock with the optic nerve at its center (-Fig. 7). In this model, the initial gross distinction is between lesions located lateral or medial to the optic nerve. For purely lateral lesions (8-10 o'clock), the lateral micro-orbitotomy is the preferred approach. If an inferior lateral extension is needed, a zygomatic osteotomy can be added (6-8 o'clock). If the lesion has superior lateral (9-1 o'clock) or intracranial extension, a frontotemporal craniotomy provides better access. For lesions located medial to the optic nerve, consideration should be given to their anterior-posterior extension before choosing the approach. Medial lesions situated in the anterior orbit (1-6 o'clock) can be accessed via the anterior medial micro-orbitotomy approach. However, medial lesions that extend posteriorly are more challenging and are ideally suited for EEA access (1-7 o'clock). In the end, the approaches should not be considered in isolation but often need to be combined to provide 360-degree access to the entire intra- and extraconal orbit. As such, the team should be comfortable with applying all approaches to offer the best option for a given pathology and patient.

\section{Conclusions}

Full, effective access to the orbit requires proficiency with a multitude of approaches. The complexity of the orbit and its relationships with surrounding structures necessitates the ability to access it from many different angles. The same general dissection and resection techniques can and should be applied throughout. Ideally, patients should be offered the best surgical approach for their pathology with anatomical relationship to the optic nerve as the primary determinant.

\section{References}

1 Yaşargil MG, Reichman MV, Kubik S. Preservation of the frontotemporal branch of the facial nerve using the interfascial temporalis flap for pterional craniotomy. Technical article. J Neurosurg 1987;67(3):463-466

2 Zabramski JM, Kiriş T, Sankhla SK, Cabiol J, Spetzler RFJ. Orbitozygomatic craniotomy. Technical note. J Neurosurg 1998;89(2): 336-341

3 Tanriover N, Ulm AJ, Rhoton AL Jr, Kawashima M, Yoshioka N, Lewis SB. One-piece versus two-piece orbitozygomatic craniotomy: quantitative and qualitative considerations. Neurosurgery 2006;58(4, Suppl 2):ONS-229-ONS-237; discussion ONS-237

4 Balasingam V, Noguchi A, McMenomey SO, Delashaw JB Jr. Modified osteoplastic orbitozygomatic craniotomy. Technical note. J Neurosurg 2005;102(5):940-944

5 Prevedello DM, Pinheiro-Neto CD, Fernandez-Miranda JC, et al. Vidian nerve transposition for endoscopic endonasal middle fossa approaches. Neurosurgery 2010;67(2, Suppl Operative):478-484

6 Hadad G, Bassagasteguy L, Carrau RL, et al. A novel reconstructive technique after endoscopic expanded endonasal approaches: vascular pedicle nasoseptal flap. Laryngoscope 2006;116(10):1882-1886

7 Cockerham KP, Bejjani GK, Kennerdell JS, Maroon JC. Surgery for orbital tumors. Part II: transorbital approaches. Neurosurg Focus 2001;10(5):E3

8 Bejjani GK, Cockerham KP, Kennerdel JS, Maroon JC. A reappraisal of surgery for orbital tumors. Part I: extraorbital approaches. Neurosurg Focus 2001;10(5):E2

9 Kennerdell JS, Maroon JC, Malton ML. Surgical approaches to orbital tumors. Clin Plast Surg 1988;15(2):273-282

10 Maroon JC, Kennerdell JS. Surgical approaches to the orbit. Indications and techniques. J Neurosurg 1984;60(6):1226-1235

11 Kiratli H, Bulur B, Bilgiç S. Transconjunctival approach for retrobulbar intraconal orbital cavernous hemangiomas. Orbital surgeon's perspective. Surg Neurol 2005;64(1):71-74

12 Takahashi R. Exposure of the optic canal. Operation 1951; 5:300-302

13 Niho S, Yasuda K, Sato T, Sugita S, Murayama K, Ogino N. Decompression of the optic canal by the transethmoidal route. Am J Ophthalmol 1961;51:659-665

14 Kennerdell JS, Amsbaugh GA, Myers EN. Transantral-ethmoidal decompression of optic canal fracture. Arch Ophthalmol 1976; 94(6):1040-1043

15 Sofferman RA. An extracranial microsurgical approach to the optic nerve. J Microsurg 1979;1(3):195-202

16 Sofferman RA. Sphenoethmoid approach to the optic nerve. Laryngoscope 1981;91(2):184-196

17 McMains KC, Kountakis SE. Contemporary diagnosis and approaches toward optic nerve decompression. Operative Techniques Otolaryngol Head Neck Surg 2006;17:178-183

18 Yoshimura K, Kubo S, Yoneda H, Hasegawa H, Tominaga S, Yoshimine $\mathrm{T}$. Removal of a cavernous hemangioma in the orbital apex via the endoscopic transnasal approach: a case report. Minim Invasive Neurosurg 2010;53(2):77-79 
24 Orbital Approaches Paluzzi et al.

19 Moe KS, Bergeron CM, Ellenbogen RG. Transorbital neuroendoscopic surgery. Neurosurgery 2010;67(3 Suppl Operative):ons16-ons28

20 Kent JS, Allen LH, Rotenberg BW. Image-guided transnasal endoscopic techniques in the management of orbital disease. Orbit 2010;29(6):328-333

21 Murchison AP, Rosen MR, Evans JJ, Bilyk JR. Endoscopic approach to the orbital apex and periorbital skull base. Laryngoscope 2011; 121(3):463-467

22 Kassam A, Snyderman CH, Mintz A, Gardner P, Carrau RL. Expanded endonasal approach: the rostrocaudal axis. Part II. Posterior clinoids to the foramen magnum. Neurosurg Focus 2005;19(1):E4

23 Kassam A, Snyderman CH, Mintz A, Gardner P, Carrau RL. Expanded endonasal approach: the rostrocaudal axis. Part I. Crista galli to the sella turcica. Neurosurg Focus 2005;19(1):E3
24 Stippler M, Gardner PA, Snyderman CH, Carrau RL, Prevedello DM, Kassam AB. Endoscopic endonasal approach for clival chordomas. Neurosurgery 2009;64(2):268-277; discussion 277-278

25 Gardner PA, Kassam AB, Snyderman CH, et al. Outcomes following endoscopic, expanded endonasal resection of suprasellar craniopharyngiomas: a case series. J Neurosurg 2008;109(1): 6-16

26 Gardner PA, Kassam AB, Thomas A, et al. Endoscopic endonasal resection of anterior cranial base meningiomas. Neurosurgery 2008;63(1):36-52; discussion 52-54

27 Gardner PA, Maroon JC, Kassam AB. Tumors of the orbit. In Winn HR (Ed.), Youmans Neurological Surgery, 6th ed. Philadelphia, PA: Elsevier Saunders; 2011:1655-1665 